\title{
Temperature Distribution in Spherical Pellets
}

by

Q. M. Anderson

May 1953

\section{/III}

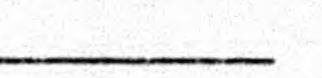

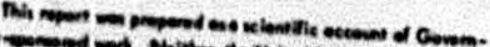

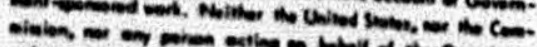

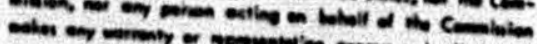

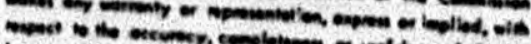

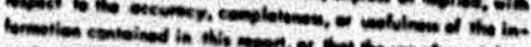

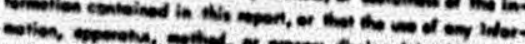

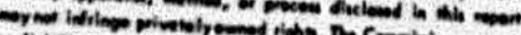

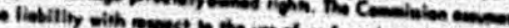

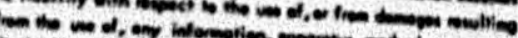
diaclowed in mis mpent.
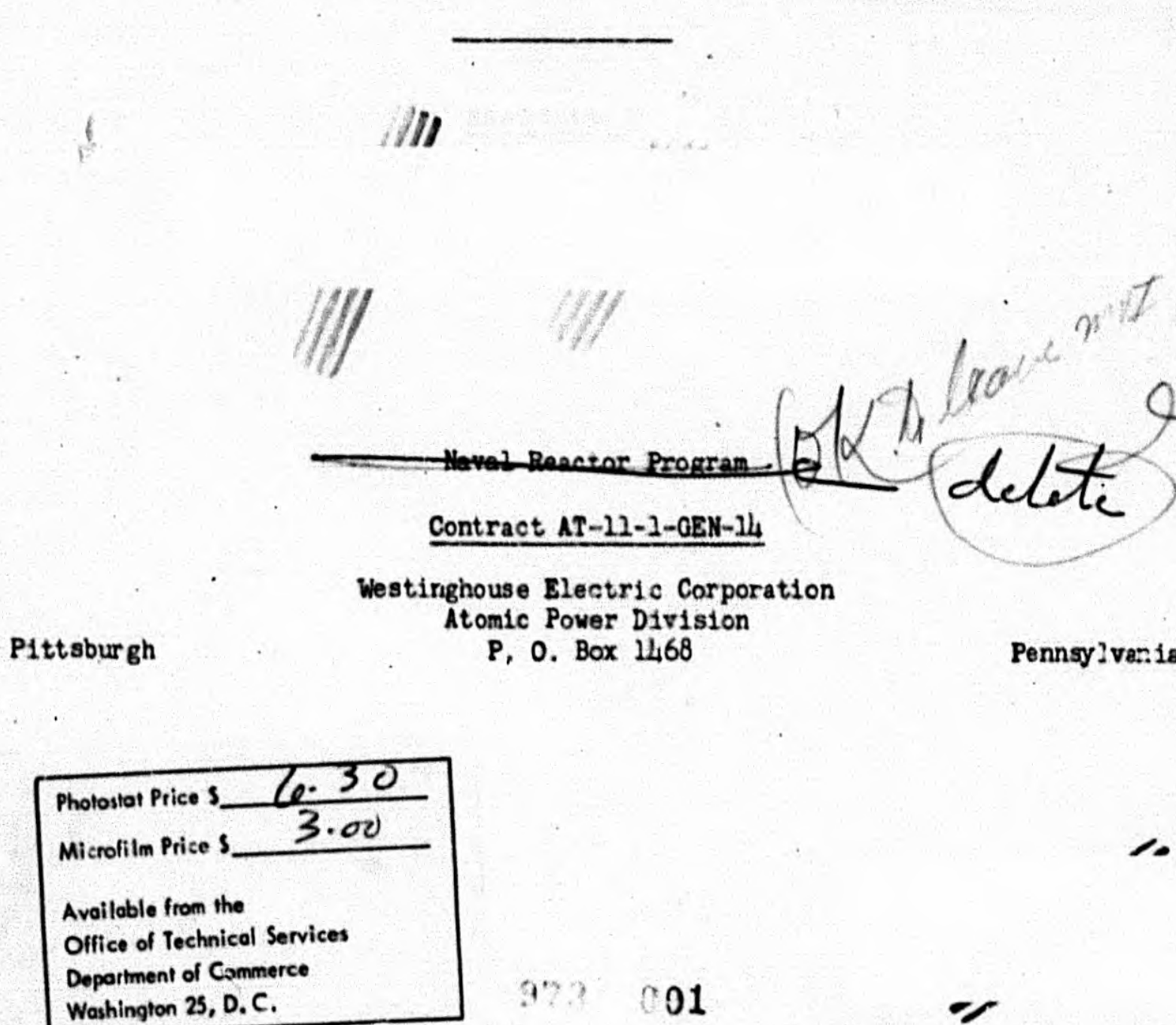

$9 \% ?$

01

Pennsy] ve:1a 


\section{Abstract}

A study is presented of the surface temperature distribution of spherical pellets in a fixed-bed, pellet-type reactor. The temperat ure near a point of contact is determined experimentally by means of an electrolytic tank analogue. An analytical approach involving the use of the IBM (CPC) calculator is described. Suggestions for fur ther st udy a re included.

$-3-$

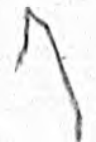




\section{Temperature Distribution in Spt ical Pellets}

\section{Introduction:}

This report sumnarizes the progress on the study of the temperature distribution in spherical pellets of a pellet type reactor. of primary importance in this investigation is the determination of the magnitude of the peak surface temperature. Hot spots occur on the surface at the points where the pellets are in contact for in this region the coolant velocity approaches zero with a resultant reduction of heat transfer.

Two general approaches are being pursued in this program. One involves an analogue technique which utilizes an electrolytic tank current flow field to simulate the heat flow fleld. The other makes use, of an analytical treatment with numerical resuits achieved by digital techniques on an IBM card programmed calculator (CPC).

The results of these two approaches are complementary in that the problems solved are somewhat different. At first it was planned to make exclusive use of the electrolytic tank because of the obvious complexity and the immense labor necessary. to achieve numerical results from analytical methods. However, while a waiting the construction of the tank considerable progress was made in developing an analytical method. Of paramount importance in furthering this phase of the work was the suggestion that the CPC calculator at the Westinghouse Anacom Laboratory be employed for the numerical calculations. 
The material presented is divided as follows:

I. Mathematical Pormulation of the Problem

II. Transformation of the Problem

III. Blectrolytic Tank Analogue

IV. Analytical Solution

v. Conclusions and Further Work

Appendix A - Fundamental Basis of the Tank Analogue Including tank design information.

Appendix B - Mathematical Analysis of the Problem and its solution in the form of an infinite series. 


\section{Mathematical Formulation of the Problem}

The problem under constderation is the deteraination of the temperaturv diatribution on the surface and in the interior of a spherical pellet in a fixed bed of Identical pellets. The pellets have a core of flasionable material covered with a sultable cladding for the purpose of minimizing corrosion, coolant flowing through the bed removes the heat generated by fissions in the core of the pellets.

Figuro 1 shows a cross section of one possible arrangement. In this Instance the matrix of spherical pellets is cubical and results in six points of contact on each sphere, Other packing arrangements may result in as many as twelve contacts.

The effects attributable to coolant variables such as velocity, viscosity and pressure are taken into account by a concept known as the $\mathrm{film}$ coefficient. The film coefficient relates the heat transfer between the surface and the coolant to the existing temperature differential. The effect of the coolant variables on the film coefficient is determined from experiment.

The data on the heat transfer coefficient for spherical pellets in a bed is rather meager. The only avallable information is that given by $W$. $H$. Denton who treats the case of eight points of contact. The data shows that for a Reynolds number of 50,000 , the case of interest here, the heat transfer coefficient rises rapldly from a zero value at the point, of contact and atteins its average value at an angle roughly five degrees removed from the contact point. Outside this region the coefficient is generally greater than the average value and at places attains a value of as much as one and a half times the average.

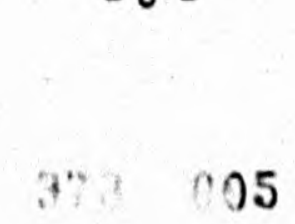


The impact of Dentont data on the course of this program has been considerable. The narrcuness of the "dead-zone" around the point of contact implies that, as a first approximation, each point may be treated separately. The temperature decreases rapldly from a peak value at the point of contact to a value which varies slowly with the slowly varying film coefficient over the non-contact regions. The large ratio of active heat transfer area to dead-zone area ensures that the points of contact do not affect each other.

A further simplification of the problem is achieved by neglecting the variation of the film coeffieient with the longitudinal angle $\phi$, of Figure 2. This assumption reduces the problem to a two dimensional ore with temperatures and fluxee dependent only on the $r$ and $\theta$ variables. Actually there is a variation of film coefficient with $\phi$, as Denton's data shows, but it is not as important as the $\theta$ variation. The first phases of this study, in fact all of the work reported here, neglect the influence of the variable.

(1)

The final step in the reduction of the problem consists of approximating the data by a step function variation of film coefficient which has zero value in a region $0 \leq \theta \leq \theta_{0}$ and a constant value in a region $\theta_{0} \leq \theta \leq \theta_{\max }$. The maximum angle, $\theta_{\max }$, should be sufficiently large so that the temperature at this point is not greatly influenced by the dead-zone angle $\theta_{0}$. In this study $\theta_{\max }$ is $90^{\circ}$. 
For non-corroded spheres the dead-zone angle is about five degrees, as explained earller. The effects of some corrosion in the hot-spot region may be approximately evaluated by varying $\theta_{0}$. It is presently contemplated to let Oo vary from five to thirty degrees. The results obtained for the larger dead-zone angles sust be viewed with considerably more skept1elsan than the small dead-zone angles aince in the former Instances the interaction effects between points of contact may invalidate the assumptions employed.

The final formulation of the problea is 1ilustrated diagramaticilly In Figure 3. Heat is generated uniformly over the core region, $r \leq a$. It flovs to the water from the sphere over the surface, $r \cdot b$, which is characterized by the filn coefficient variation of Figure 38 . 


\section{Transformation of the Problem}

The problem formulated in Part I may be transformed using the principle of superposition. As this transformation is of value in the analogue approach as well as the matheiatical analysis, it is explained here to unify the treatment as far as possible.

The basis of the transformation consists of the following observation. The zero film coefficient over the dead-zone, $0<\theta<\theta_{0}$, implies that no thermal flux can leave the sphere in this region. This boundary condition may be achieved by adding two solutions which have equal magnitude but oppositely directed fluxes at the boundary in this region.

Consider the two cases illustrated in Figure 4 . In Figure $4 \mathrm{~h}$ the $\mathrm{film}$ coefficient is independent of $\theta$. Inder this condition all of the thermal fux is radial, uniform and has a surface value $q_{0}$. The negative of the surface flux density, $-q_{0}$, is used in the second case, 1llustrated in Figure LC, to obtain zero flux density in the dead-zone when the two solutions are added. In the latter case the heat generated in the core is taken to be zero and it is this condition which makes the transformation advantageous. In the electrolytic tank analogue it is much more convenient to apply the forcing function at the boundary than uniformly over the volume. The tank and the analytical methods are set up to solve only the second part of the problem, as the radial flow case of Figure $\mathrm{LA}$ and $\mathrm{LB}$ is readily calculated. The complete solution is obtained by adding the two solutions. 
The Justification of this transformation consists of observing that the differential equations involved are linear and that the boundary conditions are properiy fulfilled.

In Figure to the current density for $\theta>\theta_{0}$ is shown dashed since it is not known in advance, but is obtained from the solution of the problem. 


\section{Blectrolytic Tank Analogue}

The fundanental basis of the current now anaiogue of the heat now fleld is explained in detall in Appendix A, The analogy stess fron the equivilence of the differential pquations governing the two phenomena.

There are two types of eurrent Nov analogues in camon use, One of these makes use of a continuous conducting mediun in which the flow of current and the resulting potentials are described by partial differential equations. The second type approximates the continuous nedium by a nesh of lumped resistors for which the currents and potentials are described by difference equations which approximate the differential equations of the electrical field. In general the fineness of the mesh structure must be such as to adequately ropresent the variables in regions of rapid change.

Barly experience on the present problem indicates that very steep temperature gradients are expected in the vicinity of the contact points. Consequently, in this region, at least, a fine mesh of resistors is required for a disnrete type of analogue. The further consideration wilch admits of varyding the dead-zone angle leads to a prohibitively large number of resistors fer this t ype of representation.

The continuous typg analogue is not restricted by the rapidity of the :

variations of the variables. Howver, there is another kind of difficulty in this case which arises from the necessity of representing the film coefficient. If electrolytes are used to represent the thermal conductivity of the core and clad materials it becomes a practical difficulty to connect a condutive material at the boundary to represent the film coefficient. 
A compromise Involving both methods is used. Electrolytes rerresent the conductivities of the core and clad regions. At the surface fixed resistors are connected for every degree of angle to represent the film coefficient. The one degree increments are believed adequate to handle the expected temperature gradients and the electrolytes eliminate the necessity of extending this fine mesh over the entire area represented.

The design is 1llustrated diagrammatically in Figure 5. The tank represents a section of the sphere of width $\Delta \phi$. It is not necessary to represent the entire sphere since the solutions are independent of $\varnothing$ under the assumptions employed. The variable thickness of the section, increasing as $r \sin \theta$, is accomplished by inclining the tank to the horizontal. A slight approximation is introduced by the fact that the sides of the tank are straight and not curved to represent the curvature of the spherical surface. This effect is minimized by keeping the angle $\Delta \phi$ small; in this instance $\Delta \emptyset=0.16 \mathrm{rad}$.

There is an insulating barrier inserted at the clad-core interface to prevent mixing of the two electrolytes which have a conductivity ratio representative of the similar thermal conductivity ratio. Connection between the two regions is achleved by using small $U$ shaped pieces of copper separated from each other and placed at one degree intervals along the interface boundary. Similar pieces are located every degree along the outer boundary for connection to the fixed resistors which represent the surface film coefficient.

Current is fed into the tank over the dead-zone region and flows out through the film coefficient resistors. The resistors are connected to a cormon $-12-$

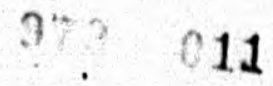


ground and the measurement of potentials relative to this ground are related to the temperature difference between the corresponding point in the thermal system and the water. To this temperature must be added the temperature resulting from the uniform flow as explained in Part II.

The results of tank measurements are plotted in Figure 7 and show the functional dependence of the dimensionless ratio

$$
\frac{K_{c}}{b_{T}} \frac{T(r=b)}{90}=f(\theta)
$$

for several values of $\theta_{0}$. The other dimensionless parameters,

$$
\begin{gathered}
\pi_{1}=\left(\frac{K_{c}}{\mathrm{~K}_{\mathrm{m}}}\right)=2 ; \pi_{2}=\left(\frac{\mathrm{b}}{\mathrm{a}}\right)=1.25 \\
\pi_{6}=\frac{h_{\mathrm{f}}}{\mathrm{K}_{\mathrm{c}}}=15.1
\end{gathered}
$$

were held constant during the course of the experiment. The symbols are defined in the nomenclature at the end of the report.

An important inference to be drawn from Figure 7 is the linear dependence of hot spot surface temperature on the dead zone angle $\theta_{0}$. 


\section{Analytical Solution}

- The heat flow problem in spherical pellets formulated in Parts I and II is a boundary value problem. There are a variety of analytical methods which have been developed to treat such problems. In general the methods involve series or iterative methods of computation which have the disadvantage of being vexy time consuming. The advent of modern machine methods has greatly reduced this limitation and has made these techniques available for the solution of practical problems.

Unfortunately the analytical methods deal mostly with problems which specify either the potential or the flow variable on the boundary. In the present instance the specification over part of the boundary is in the form of a film coefficient which is the ratio of heat flux to temperature differential across the sphere-water interface, A straightforward approach to this problem which would yield solutions in a reasonable time, even with machine methods, has not been found. However, a method which makes use of both series and iterative techniques has been devaloped that appears to offer promise of an adequate solution.

The approach is to change the problem to one of the standard types. In this case the thermal flux over the surface of the sphere is specified. The result ing temperature distribution is then determined by a series method. Comparison of the assumed thermal flux and the calculated temperature indicates the manner in which the flux assumption should be modified to achieve the constant film coefficient condition. 
Figure 6 shows the assumed thermal flux distribution. The heat flow density over the region near the point of contact is uniform and directed into the sphere as required by the boundary condition. As a first approximation the flux density is assumed constant over the rest of the sphere and may be modified in successive calculations to conform with the resulting temperat ure distribution.

In Appendix B it is shown that the temperature distribution within the sphere may be represented by

$$
T_{c}-T_{0}=\frac{q_{0 b}}{k_{c}} \sum_{n=1}^{\infty}\left[\left(\frac{r}{b}\right)^{2 n} A_{2 n}+\left(\frac{r}{b}\right)^{-(2 n+1)} B_{2 n}\right] P_{2 n}(\theta) ; a \leq r \leq b
$$

$$
T_{m}-T_{0}=\frac{90 b}{k_{c}} \sum_{n=1}^{\infty}\left(\frac{r}{b}\right)^{2 n} C_{2 n} \quad P_{2 n}(\theta) ; \quad 0 \leq r \leq a
$$

The constants $A_{2 n}, B_{2 n}$ and $C_{2 n}$ are determined from the equivalence of the temperature and thermal flux at the clad-meat interface and from the impressed thermal flux at the outer boundary. The equations are

$$
\begin{aligned}
& c_{2 n}=\left(\frac{a}{b}\right)^{2 n} A_{2 n}+\left(\frac{a}{b}\right)^{-(2 n+1)} B_{2 n} \\
& \left(\frac{a}{b}\right)^{2 n}\left[\left(\frac{k_{c}}{k_{m}}\right)-1\right] A_{2 n}-\left(\frac{a}{b}\right)^{-(2 n+1)}\left[\left(\frac{k_{c}}{k_{m}}\right)\left(1+\frac{1}{2 n}\right)+1\right] B_{2 n}=0 \\
& A_{2 n}-\left(1+\frac{1}{2 n}\right) B_{2 n}=-\left(1+\frac{1}{4 n}\right) \frac{2}{q_{0}} \int_{0}^{1} q_{B}(\mu) P_{2 n}(\mu) d
\end{aligned}
$$

$$
\mu=\cos \theta
$$


The functions $P_{2 n}(\mu)$ are the Legendre polynomials of the first kind which are orthogonal over the range $0 \leq_{\mu} \leq 1$. $q_{8}(\mu)$ is the impressed $f l u x$ distribution.

The series of Equations (3) and (4) omit the non-varying terms corresponding to the zero values of the index $n$, although these terms satisfy the differpntial equations. The reason for this omission is that the corresponding constants $A_{0}, B_{0}$ and $C_{0}$ are indeterminate for a boundary condition in terms of thermal flux density. These constants are determined by accounting for the temperature drop in the surface film.

The temperature on the surface, $\mathbf{r}=\mathrm{b}$ is obtained from Equation (3) by summing the series at $r=b$.

$$
T_{c}-T_{o} /\left(\frac{q_{0 b}}{K_{c}}\right)=\sum_{n=1}^{\infty}\left[A_{2 n}+B_{2 n}\right] P_{2 n}(\theta)
$$

The calculation of the temperature distribution for four cases of cead zone angle, $\theta_{0}$, is being performed at the Anacom Laboratory on the IBM CPC equipment. Results of this calculation will indicate the temperature for ten degree increments of $\theta$ for each of the four $\theta_{0}$ 's.

The next approximation to the case of constant film coefficient is obtained from these results by perturbing the heat flux assumption in the required direction. The machine program must be modified to perform an iteration since the definite integral of Equation (7) changes with the new flux distribution. For the flux distribution shown in Figure (7), the 
evaluation was greatly facilitated by the fact that $q_{8}(\theta)$ is constant in each of the two regions. In this case the integral may be evaluated from the expression

$$
\int P_{n}(\mu) d \mu=\frac{P_{n+1(\mu)}-P_{n-1(\mu)}}{2 n+1}
$$

In which only the values of the legendre polynomials at $\theta_{0}$ are required. These values may be computed with the aid of the recurrence formula,

$$
(n+1) P_{n+1}+n P_{n-1}=(2 n+1), P_{n}
$$

In the first iteration the evaluation of the definite integral may be accomplished by varying the assumed thermal flux in a series of steps so that use may be made of Equation (10). At the present time it is not planned to perform this iteration owing to the cost of the computation. Since the problem as formulated in Part I is in itself only an estimate as to what will take place in the bed, it seems inappropriate to go to great expense to represent this approximation in an exact manner. Rather these initial calculations may be viewed as complementing the electrolytic tank work. They provide additional bounds on the temperature variations which may occur. 


\section{Conclusions and Further Work}

The surface temperature distribution of a spherical pellet in the vicinity of a point of contact has been determined experimentally by means of an electrolytic tank analogue. The results show the variation of surface temperature with the spherical coordinate $\theta$ for several different values of dead zone angle $\theta_{0}$. It is assumed throughout that the heat transfer coefficient is zero in the dead zone and constant outside this region.

Similar results are expected shor tly from an analytical method which makes use of a series expansion technique. The necessary numerical calculations are being performed by the Westinghouse Anacom Laboratory on the IBM, CPC calculator. The results achieved analytically will differ from the electrolytic tank results and should be somewhat more pessimistic. The reason for this is that the analytical method is an iterative one and which needs several iterations to converge. At present only the first approximation is planned. The results of this study may be expanded in several directions if it is desired. The electrolytic tank as designed can readily be used for different conductivity ratios and wi th relatively minor modifications for different ratios of clad to meat radil. The analytic technique is even more flexible in these regards since to change ratios one merely replaces an old ratio with a new one and repeats the computation.

The problem of modifying the film coefficient to conform more closely with the experimental data is one which may bo worthy of attention. The present approach assuming the coefficient to be zero in the dead zone 
and constent outside this region is undoubtedly pessimistic. However, if the temperatures as computed thereunder are too large it may be necessary to explore the possibility of refining this assumption. 


\section{Nomenclature}

$T_{m}$ - Temperature in the meat or core - of

$\mathbf{T}_{\mathrm{c}}$ - Temperature in the clad - $\mathbf{O}_{\mathbf{F}}$

$T_{0}$ - Component of temperature independent of $r$ and $\theta-O F$

$k_{m}$ - Meat conductivity - Btw/hr sq ft ${ }^{G} / \mathrm{ft}$.

$k_{c}$ - Clad conductivity - Btu/hr sq ft ${ }^{0} \mathbf{F} / \mathrm{ft}$.

h - Film coefficlent - Btu/hr sq ft ${ }^{\circ} \mathrm{F}$

qv - Heat generated per unit volume - Btw/hr cu ft.

q - Thermal flux density - Btu/hr sq ft.

$q_{0}$ - Constant surface thermal flux density corresponding to flux case of uniform film coefficient. Btw/hr sq $\mathrm{ft}$.

$q_{s}$ - Surface Rux density - Btu/hr sq ft.

V - Voltage in analogue - volts

$V_{\mathrm{a}}$ - Voltage applied to the current density source resistors.

J - Blectrical current density - Amps/sq ft.

$J_{v}$ - Volumetric production of current - amps/cu ft.

$\sigma_{m}-$ Blectrical analogue conductivity of the meat - mhos/ft.

$\sigma_{c}$ - Blectrical analogue conductivity of the clad - mhos/ft.

$\lambda$ - Electrical analogue conductivity of the $\mathrm{flm}$ - mhos/sq $\mathrm{ft}$.

aT - Radius of meat in thermal system - ft.

b

a. - Radius of meat in electrical system - ft.

$b_{0}$ - Radius of clad in electrical system - ft.

R1 - Resistance associated with the $i^{\text {th }}$ connection at the surface. (represents the film coefficient - ohms). 
$R_{s_{1}}$ - Current density source resistors.

$r, \theta, \emptyset$ - Spherical coordinate vaziables.

$-21-$

$9 * 020$ 
Appendix A

1. Basis of Current Flow Analogue

The possibility of using a current flow field to represent a thermal heat flow field stems from the equivalence of the differential equations governing the two cases.

In the thermal case
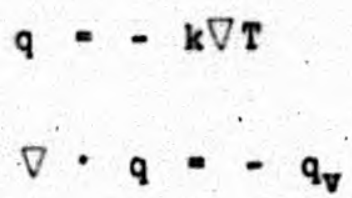

In the electrical syutex

(13)

$$
\begin{aligned}
& \mathrm{J}=-\sigma \nabla \mathrm{V} \\
& \nabla \cdot \mathrm{J}=\mathrm{J}_{\mathrm{V}}
\end{aligned}
$$

The correspondance between these systems of equations forms the basis of the analogy. The $j_{v}$ term is not used in the analogue but is included here to show what is required if the transformation described in Part II is not employed.

The mere correspondance of the differential equations of an analogue and the system it represents is not sufficient in itself to insure that the systems are completely equivalent. It is also necessary that the particular numerical values of the dimensionless ratios chracterizing the systems be made equal. 
The present systems may be characterized by the following cimensionless ratios.

$$
\begin{array}{ll}
\text { Thermal } & \text { Electrical } \\
\pi_{1 \mathrm{~T}}=\left(\mathrm{k}_{\mathrm{c}} / \mathrm{k}_{\mathrm{m}}\right) & \pi_{10}=\left(\sigma_{\mathrm{c}} / \sigma_{\mathrm{m}}\right) \\
\pi_{2 \mathrm{~T}}=\left(\mathrm{b}_{\mathrm{T}} / \mathrm{a}_{\mathrm{T}}\right) & \pi_{2 \mathrm{e}}=\left(\mathrm{b}_{\mathrm{e}} / \mathrm{a}_{\mathrm{e}}\right) \\
\pi_{3 \mathrm{~T}}=\theta_{\mathrm{T}} & \pi_{30}=\theta_{\mathrm{e}} \\
\pi_{4 \mathrm{~T}}=\theta_{0} & \pi_{40}=\theta_{0} \\
\pi_{5 \mathrm{~T}}=\left(\mathrm{k}_{\mathrm{c}} / \mathrm{b}_{\mathrm{T}}\right)(\mathrm{T} / \mathrm{q}) & \pi_{5 e}=\left(\sigma_{\mathrm{c}} / \mathrm{o}_{\mathrm{e}}\right)(\mathrm{V} / \mathrm{j}) \\
\pi_{6 \mathrm{~T}}=\left(\mathrm{h} \mathrm{b} / \mathrm{k}_{\mathrm{c}}\right) & \pi_{6 \mathrm{e}} \cdot\left(\lambda_{\mathrm{b}} / \sigma_{\mathrm{c}}\right)
\end{array}
$$

For the analogue to represent the physical system it is necessary that

$$
\pi_{\mathrm{rr}}=\pi_{\mathrm{ne}} ; \mathrm{n}=1,2, \ldots 6 .
$$

\section{Representation of the Film Coefficient}

As explained earlier the fllm coefficient is represented by fixed resistors connected to the outer boundary of the tank at one degree intervals. The numerical value of a resistor is determined from the equivalence of the $\pi_{6}$ 's and the size of the elemental area served by ne point of connection. The resistance is

$$
B_{1}: \frac{1}{\lambda A_{1}}=\frac{1}{\lambda_{b_{e}^{2} \sin \theta_{1} \Delta \theta \Delta \phi}},
$$




$$
\theta_{1}: 5-1 / 2 ; 6-1 / 2 ; \quad \ldots 89-1 / 2 \text {. }
$$

and substituting for $\lambda$ from Equation (山)

$$
\lambda=\frac{c_{c}}{b_{e}} \pi_{6}
$$

there results

$$
R_{1}=\frac{1}{\pi_{6} \sigma_{c} b_{e} \sin \theta_{1} \Delta \theta \Delta \phi} \text { ohms. }
$$

The final selection of the resistance depends on the choice of $c_{c}$ and the value of $\pi_{6}$. For the present problem

$$
\pi_{6}=15.1 ; \quad b_{e}=25 \mathrm{in} .
$$

Instead of conductivity it is more common in electrical sys tems to use the reciprocal quantity, resistivity, and the value chosen for the clad resistivity, $P_{c}$, is.

$$
P_{c} \cdot \frac{1}{c_{c}}=250 \mathrm{ohm}-\mathrm{cm}
$$

\section{The resulting expression for the film resistances is}

(18)

$$
R_{1}=\frac{93.6}{\sin \theta_{1}} \text { ohms. }
$$

The resistances for $\theta_{1}=5-1 / 2$ to $29-1 / 2$ are connected to the tank through switches so they may be disconnected at $t$ imes when it is desired to extend the dead-zone above $5^{\circ}$.

$$
-24-
$$




\section{Source Resistors}

The constant current density which is supplied over the dead zone angle is achioved by using a voltage source and large series resistors connected to the tank at one degree intervals. The following resistance values

$$
R_{B_{1}}=\frac{(200)(93.6)}{\sin \theta_{1}}=\frac{18,720}{\sin \theta_{1}} \text { ohms; } \theta_{1}=1 / 2 ; 1-1 / 2 ; \ldots 29-1 / 2
$$

reduce the source voltage by a factor of about one hundred and adequately meet the currsat density source condition. The switches which disconnet the film coefficient resistors at the same time connect additional source resistance when the dead zone is extended above five degrees.

4fophaputing the value of $\pi_{5}$ from measured analogue voltages it is convenient to express the dead-zone current density, Jo, in terms of the sore readily measured applied voltage.

Assuming the tank voltage small compared to the applied voltage, the applied current density is

$$
\text { Jo }=\frac{\lambda}{200} v_{a}
$$

where the factor of two hundred is the one used in computing the source resistors.

From Equation (14)

$$
\lambda=\frac{\sigma_{c}}{b_{e}} \pi_{6}
$$


and upon substituting into the expression for $j_{0}$ yields

(22)

$$
J_{0}=\frac{\sigma_{c}}{b_{0}} \frac{\pi_{6}}{200} \nabla_{a} \text {. }
$$

Upon this result $\pi_{5}$ from Bquation (14) is reduced to the following form

$$
\pi_{5}=\frac{200}{\pi_{6}} \frac{\mathrm{v}}{\nabla_{\mathrm{a}}}=13.2\left(\frac{\mathrm{V}}{\nabla_{\mathrm{a}}}\right) \text {. }
$$




\section{Appendix B}

Analytical Solution of the Heat Flow Problem in a Sphere when the Boundary Condition is Specified in the Form of a Thermal Flux.

The differential equation governing the steady flow of heat in a conducting region is known as Laplace's equation. In spherical coordinates the general solution of this equation, for fields which are independent of $\varnothing$ may be expressed in terms of an infinite series of the form

$$
T=\sum_{n=0}^{\infty}\left[A_{n}^{\prime} r^{n}+B_{n} r^{-(n+1)}\right] P_{n}(\theta)
$$

where $r$ and $\theta$ are the spherical coordinates and $P_{n}(\theta)$ are the Legendre polynomials of the first kind of order $n$. To be perfectly general the solution should include the functions $Q_{n}(\theta)$, the Legendre polynomials of the second kind. However, all of these functions are infinite for $\theta=0$ and are therefore not admissible in this problen.

The Legendre polynomials are orthogonal over the range from 0 to $\pi$. It is therefore comvenient to imagine that the $\theta$ variation is extended from $\pi / 2$ to $\pi$ and that in this region the film coefficient is symmetrical about $\pi / 2$. This in no way affects the solution in the interval from 0 to $\pi / 2$ but permits the use of the $\boldsymbol{\alpha}$ thogonal properties of the legendre polynomials.

The symmetry about $\theta \cdot \pi / 2$ eliminates the odd-order polynomials from the solution. Separate expressions for the temperature ere required for the core and clad regions because of the different conductivities. It 1 s convenient to change the constants $A_{h}$ and $B_{h}$ slightly to make them dimensionless. Making these changes Equation (23) takes the form

$$
-27^{\prime}-
$$


(24)

$$
T_{c}-T_{0}=\frac{g_{0 b}}{k_{c}} \sum_{n=1}^{\infty}\left[A_{2 n}\left(\frac{r}{b}\right)^{2 n}+B_{2 n}\left(\frac{r}{b}\right)^{-(2 n+1)}\right] P_{2 n}(\theta)
$$

$$
T_{m}-T_{0}=\left(\frac{q_{0} b}{k_{c}}\right) \sum_{n=1}^{\infty} C_{2 n}\left(\frac{r}{a}\right)^{2 n} P_{2 n}(\theta)
$$

where $T_{0}$ is the contribution from the zero term of the series, as explained earlier. The expression for the temperature in the meat does not involve the reciprocal $r$ terms since they are infinite at the origin.

The constants are evaluated from the boundary conditions at

$$
r=a \quad T_{m}=T_{c} \quad k_{m} \frac{\partial T_{m}}{\partial r}=k_{c} \frac{\partial T_{c}}{\partial r}
$$

and at $r \cdot b$

$$
\begin{aligned}
q_{s} & =-k_{c} \frac{\partial T_{c}}{\partial r} \\
& =-q_{0} \sum_{n=1}^{\infty}\left[2 n A_{2 n}-(2 n+1) B_{2 n}\right] P_{2 n} \quad(\theta)
\end{aligned}
$$

which upon substitution from Equations $(24)$ and $(25)$ yield the expression given in Equations $(5),(6)$ and $(7)$ : 


\section{Bibliography}

1. W. H. Denton, The Heat Tre nsfer and Flow Resistance for Fluid Flow Through Randomly Packed Spheres." p. 371. Published by The Institution of Mechanical Engineers. Proc, of "General Discussion on Heat Transfer" 11-13th September 1951. 


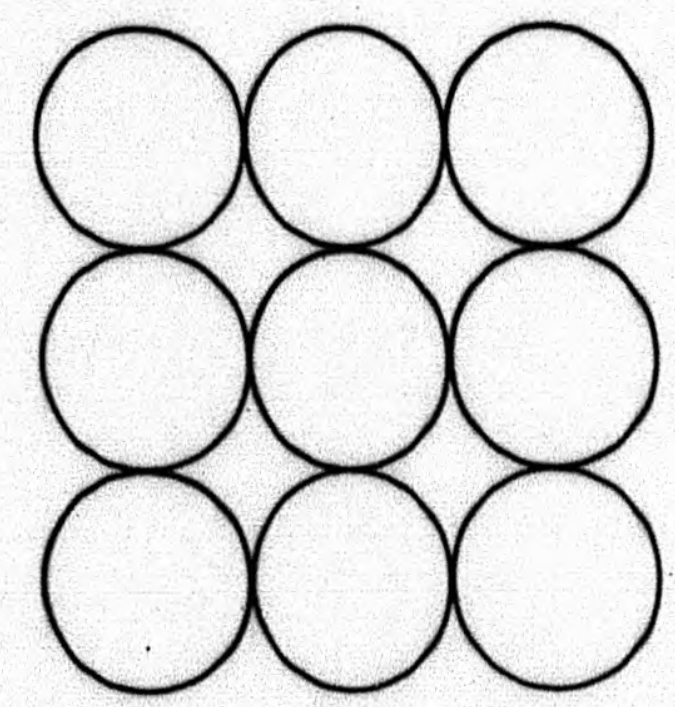

CROSS SECTION OF A PELLET BED FIGURE I

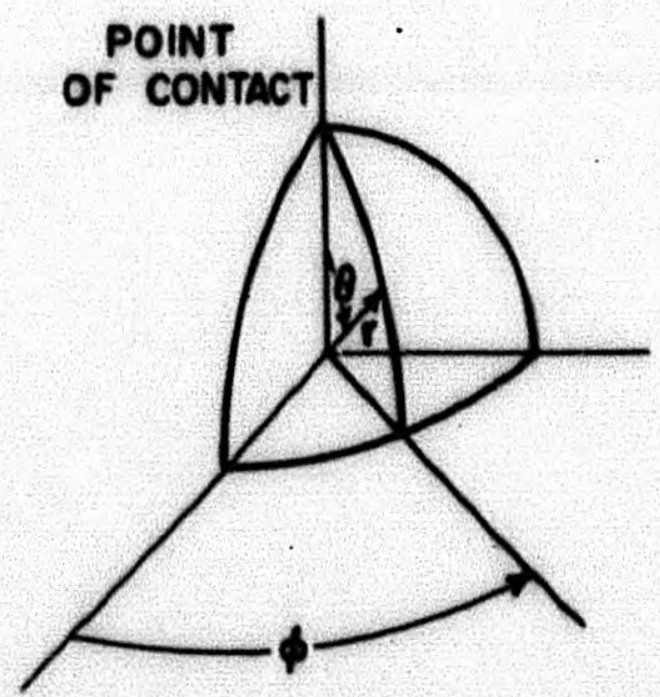

SECTION OF A SPHERICAL PELLET FIGURE 2

$$
\begin{array}{r}
-30- \\
912 \quad 029
\end{array}
$$




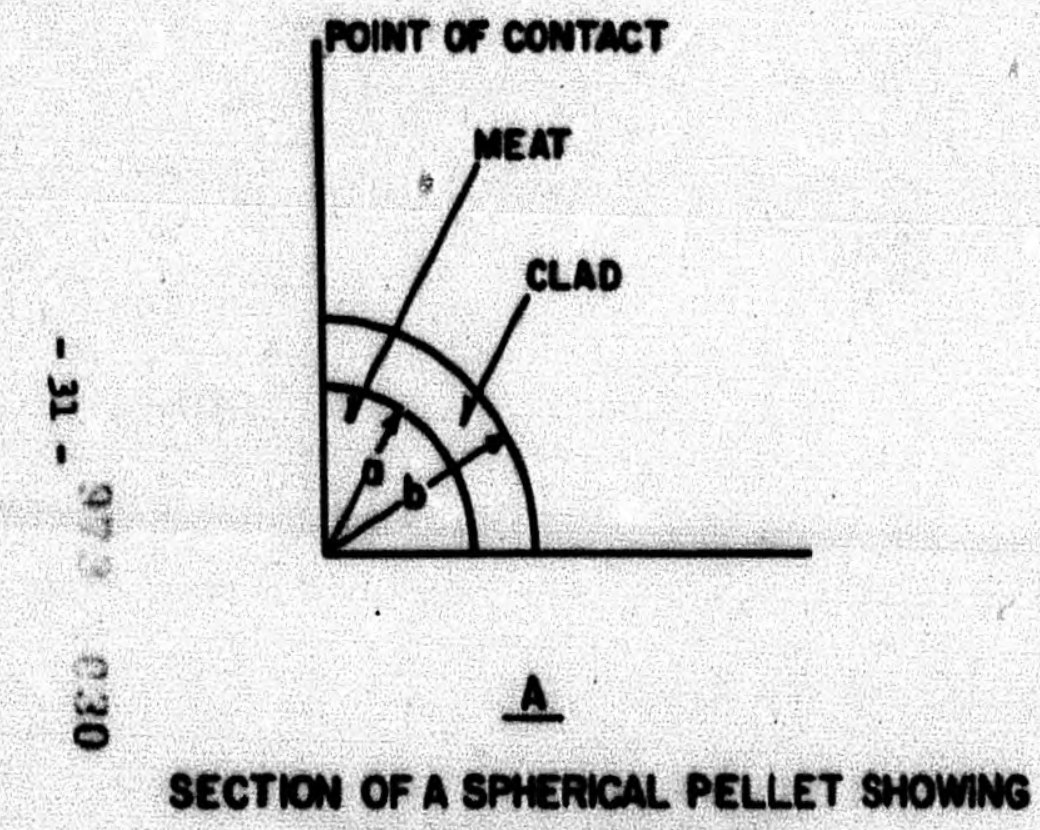

vEAT AD CLAD REGIONS

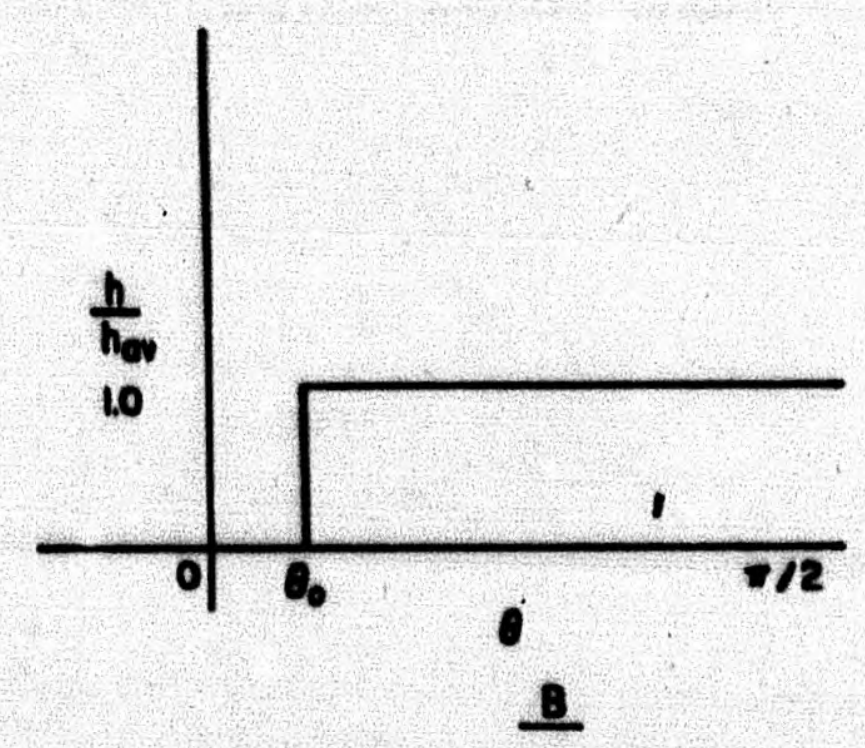

ASSUMED VARIATION OF FILW COEFFICIENT

FIGURE 3 


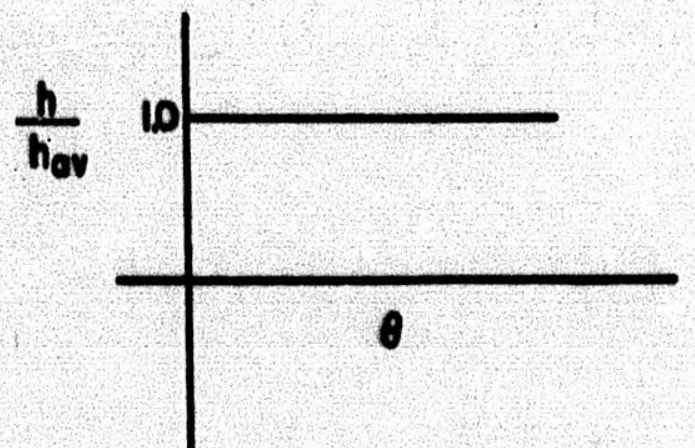

(A)

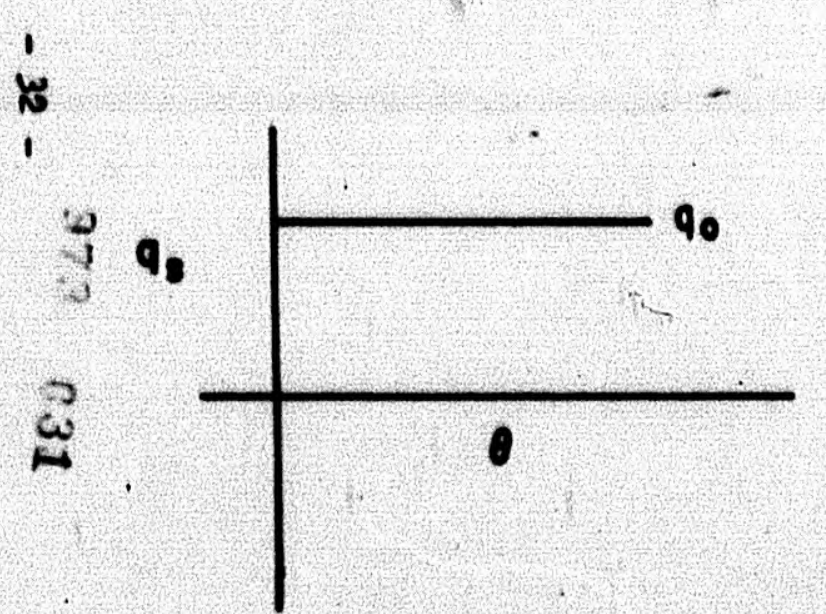

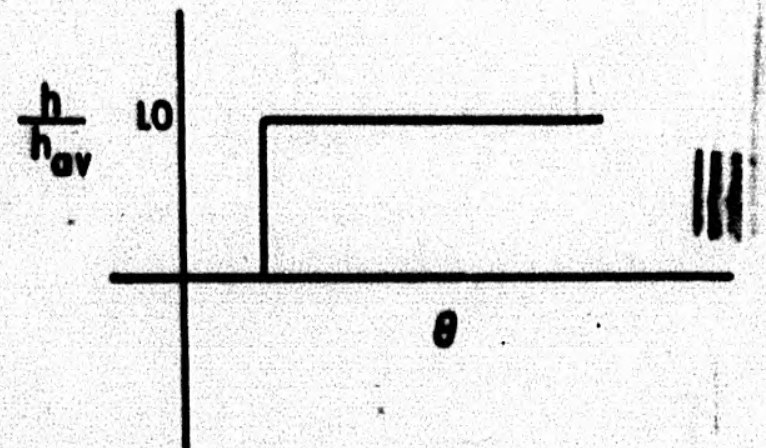

(c)

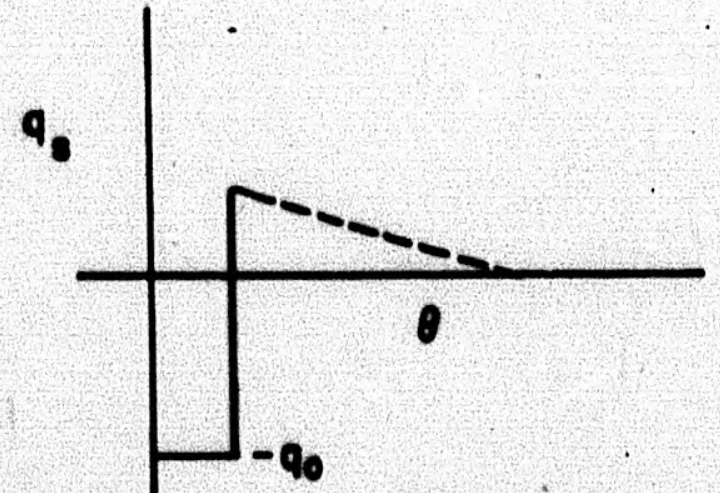

(D)

(B)

APPLICATION OF SUPERPOSITION TO THE HEAT FLOW PROBLEM OF PART I

FIGURE 4 


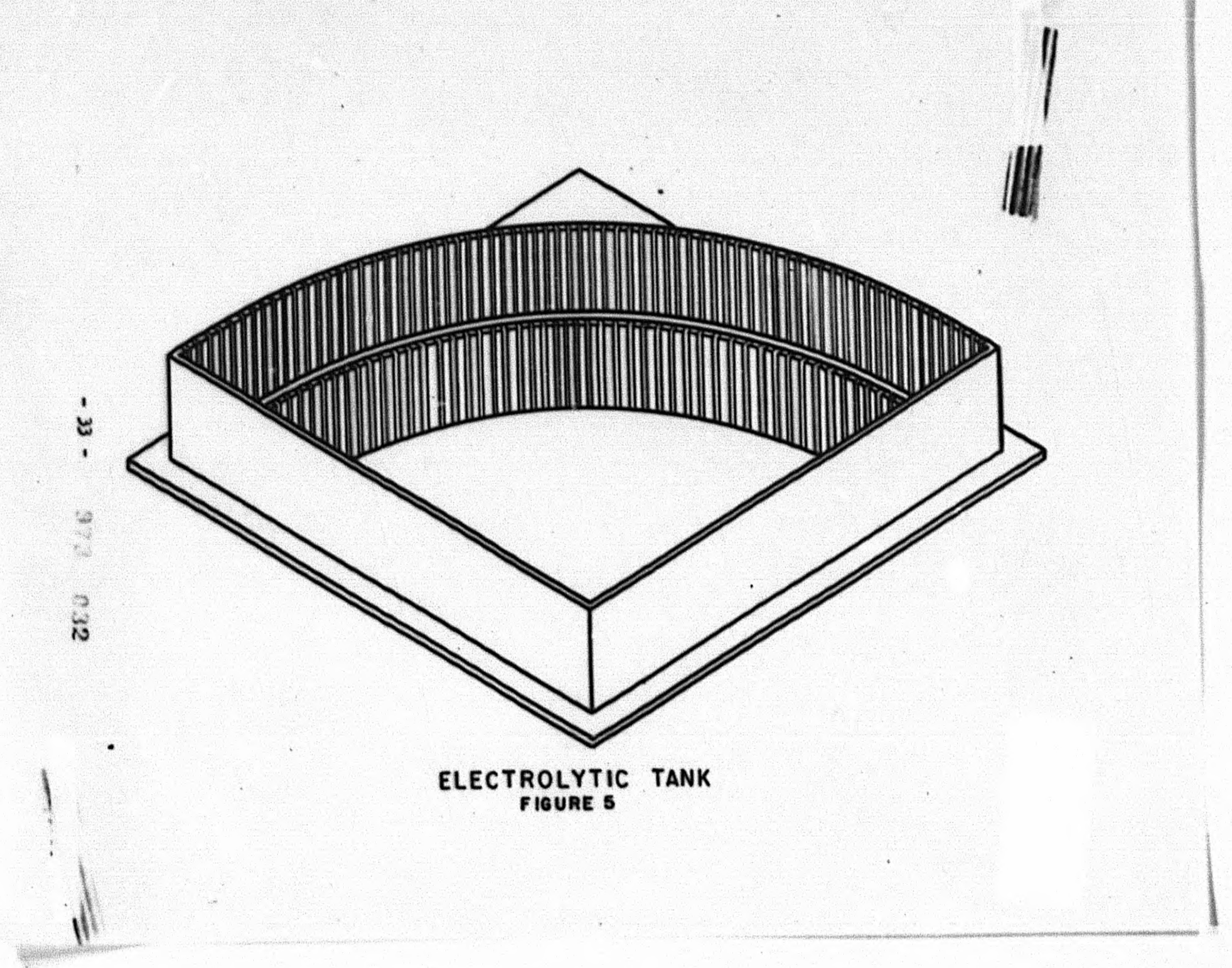




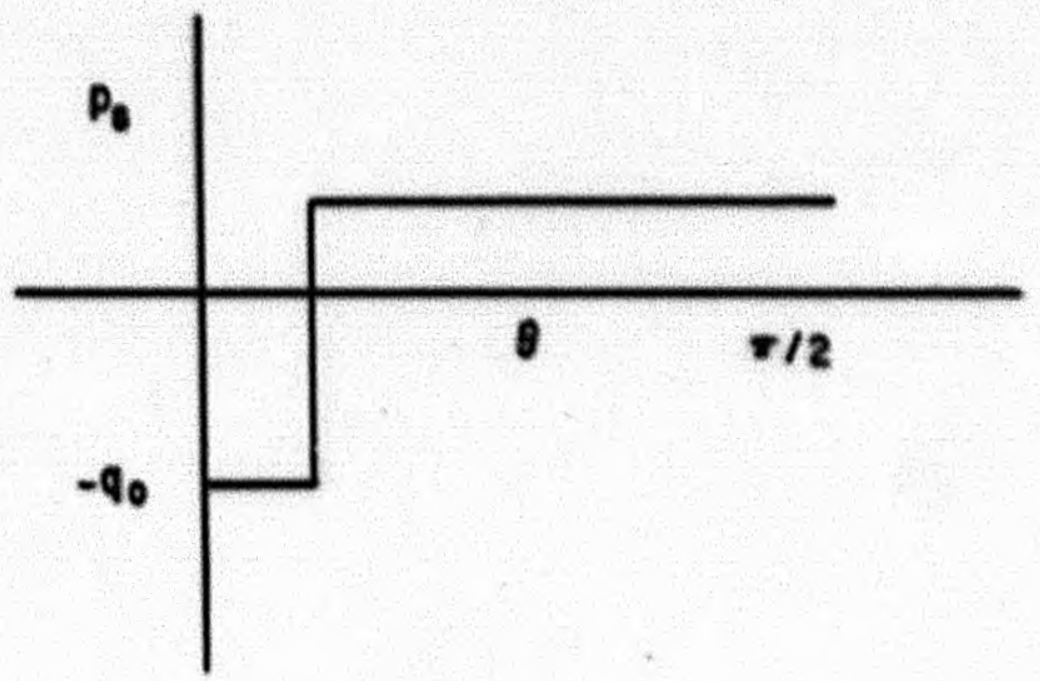

SURFACE FLUX DENSITY ASSUMED AS A FIRST APPROXIMATION IN THE ANALYTICAL METHOD

FIGURE 6

-34 -

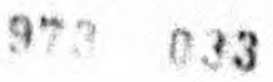


ב. plot of the owensioness ratio

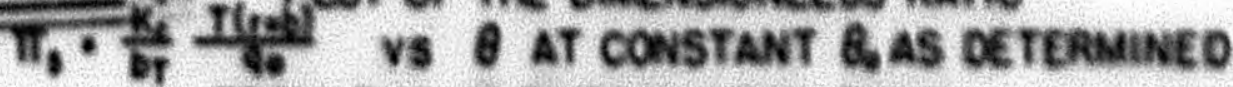
FROM THE ELECTROLYTIC TANK
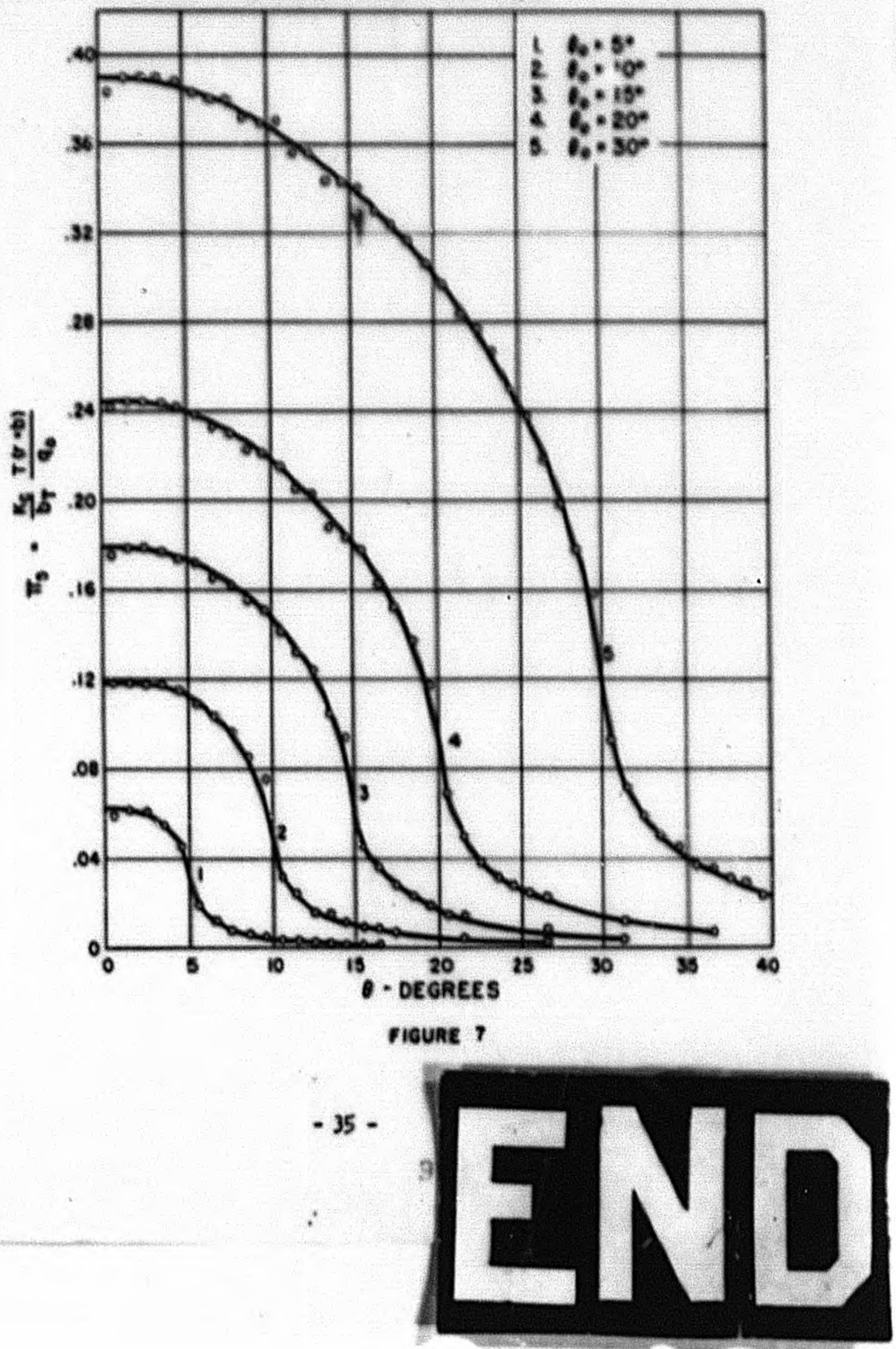